\title{
Que peut apporter l'analyse isotopique en flux continu à la recherche et aux utilisations biomédicales ?
}

\author{
J.L. Brazier et W. Elbast \\ Chaire Médicaments et Grossesse, Université de Montréal Faculté de Pharmacie, CP 6128 succursale Centre-ville, Montréal H3C 3J7, \\ Québec
}

\begin{abstract}
Isotope ratio mass spectrometry and especially continuous flow isotope ratio mass spectrometry (CF-IRMS) expand rapidly in various fields including geochemistry, oil and earth sciences, environment, authentication of organic compounds. The last area overtaken by CF-IRMS is that of biomedical research. This paper tries to answer the question: what can CF-IRMS provide to biomedical research? The answer relies on three main series of advantages: advantages provided by stable isotope labelling, advantages provided by the high analytical performances of IRMS itself, advantages provided by hyphenation of IRMS with dedicated sample preparation devices. These advantages are discussed and illustrated with numerous examples. The development of non invasive methods applied to medical diagnosis and their applications in gastro-enterology are widely presented as well as the potentiality of IRMS to allow the use very low levels of labelling and naturally enriched tracers for metabolic studies.
\end{abstract}

\section{Introduction}

L'analyse isotopique et plus particulièrement l'analyse isotopique en flux continu s'est développée rapidement ces dernières années dans les champs d'application représentés par la géochimie, les sciences de la terre, les sciences de l'environnement, le domaine pétrolier. Le domaine de l'authentification de substances organiques quant à leur origine ou au respect de leurs certifications est un secteur en plein essor et dans certains pays, les réglementations de contrôle et des fraudes préconisent l'utilisation de ces technologies aux fins de certification. Les domaines de la recherche et des utilisations biomédicales et cliniques ont mis plus de temps pour recourir à l'analyse isotopique en flux continu pour des raisons de formation initiale des chercheurs, de diffusion de l'information et en raison d'impératifs particuliers liés aux études et recherches biomédicales. Le vaste domaine d'investigation d'abord ouvert par l'analyse des isotopes du carbone s'élargit de plus en plus avec l'accès maintenant possible aux isotopes de l'azote, de l'oxygène, du soufre et de l'hydrogène. Ainsi peut on essayer d'apporter une réponse actuelle à la question : que peut apporter l'analyse isotopique en flux continu (CF-IRMS - continuous flow isotope ratio mass spectrometry) à la recherche et aux utilisations biomédicales ?

L'analyse isotopique en flux continu permet d'utiliser les avantages procurés par (i) les isotopes stables, (ii) l'analyse isotopique elle-même, (iii) les méthodes de couplage avec des systèmes de séparation hautement performants et ceci pour répondre aux impératifs spécifiques de la recherche biomédicale et des utilisations cliniques.

\section{Avantages des isotopes stables}

Le premier avantage des isotopes stables réside dans l'utilisation de molécules marquées par ces isotopes non radioactifs. Celles ci constituent d'excellents traceurs et marqueurs biologiques dénués de radioactivité. Il n'y a donc pas à craindre de dangers liés à l'utilisation de molécules radiomarquées. Il n'y a pas à se conformer à des restrictions légales ou réglementaires particulières régissant l'emploi de ces molécules. Ces molécules permettent ainsi de concevoir des protocoles cliniques avec un niveau d'éthique extrêmement élevé. Enfin l'utilisation des molécules marquées par des isotopes stables ne pose pas de problèmes pour les rejets et n'a aucun impact néfaste sur l'environnement. Un autre avantage particulier des isotopes stables réside dans le marquage lui-même. En effet celui-ci est quantitatif et réalisé sur des sites moléculaires précis. Ce dernier point est particulièrement intéressant pour conduire des études de métabolisme, de turn over, de réincorporation ou recyclage de fragments moléculaires au cours de cycles métaboliques. Enfin, et c'est le cas plus particulièrement pour les isotopes ${ }^{13} \mathrm{C}$ et ${ }^{15} \mathrm{~N}$, le marquage n'induit pas de toxicité ni d'embryotoxicité intrinsèque. De plus les effets isotopiques au cours de processus macroscopiques comportant plusieurs étapes (déterminations de constantes de vitesse de transfert, de paramètres pharmacocinétiques, description de voies métaboliques) sont négligeables. Ce n'est pas le cas pour le deutérium qui lui, peut induire des effets isotopiques majeurs $[1,2]$. 


\section{Avantages de l'analyse isotopique}

Les développements technologiques des dernières années ont mis sur le marché des appareillages permettant les déterminations d'enrichissements isotopiques et les mesures de rapports isotopiques avec très hautes précision, exactitude, sensibilité. La détermination des isotopes du carbone, de l'azote, mais aussi de l'hydrogène et du soufre peut être réalisée par analyse isotopique en flux continu à partir de très faibles quantités d'analytes et avec des niveaux de marquage très bas. Le couplage de l'analyse isotopique avec des méthodes de séparation chromatographiques, des analyseurs élémentaires ou des systèmes de pyrolyse permettent maintenant d'analyser toutes sortes d'échantillons : solides, liquides ou gazeux y compris des mélanges de haute complexité à partir de micro échantillons.

\section{Impératifs de la recherche biomédicale et clinique}

Le premier des impératifs liés à la recherche biomédicale et clinique réside dans le souci permanent de travailler avec des échantillons les plus petits possibles. Beaucoup d'études métaboliques (en nutrition ou pharmacologie) nécessitent de travailler avec des niveaux de marquage faibles afin de ne pas perturber les dimensions des pools métaboliques. Enfin, la recherche clinique et le diagnostic privilégient les méthodes les moins pénibles pour le patient et si possible des méthodes totalement non invasives. De nombreuses études étant des études cinétiques, il faut envisager le recueil de très nombreux échantillons sur des nombres parfois élevés de sujets ce qui amène à devoir analyser de très nombreux échantillons. De plus, afin de décrire au mieux et avec le plus de précision les processus cinétiques afin de les modéliser, les protocoles requièrent de courts intervalles de temps entre les recueils d'échantillons et des temps d'échantillonnage courts. Les études métaboliques, quant à elles, requièrent une spécificité des sites de marquage afin de pouvoir suivre le devenir de sites moléculaires précis tout au long des voies métaboliques étudiées et de pouvoir calculer des taux de recyclage et de réincorporation au cours de cycles métaboliques. Cette spécificité des marquages est essentielle pour les études de mécanismes. Enfin la recherche clinique demande de plus en plus l'usage de traceurs non radioactifs toutes les fois que cette alternative est possible. Cela est la condition stricte pour les études conduites dans des populations à risque ou des populations particulièrement protégées : femmes enceintes, nouveau-nés, enfants.

\section{Comment la CF - IRMS peut elle répondre aux impératifs biomédicaux et cliniques?}

Le développement de nouvelles techniques, leur amélioration induisent une amélioration des protocoles et des résul- tats en permettant le développement de méthodes non invasives et sures, en autorisant l'accès à des populations de patients particulièrement protégées, en rendant possible des protocoles jusque là non réalisables avec d'autres méthodologies.

\section{Les tests respiratoires au ${ }^{13} \mathrm{C}$ - nouveaux outils de diagnostic}

Le premier domaine dans lequel le développement et les améliorations sont spectaculaires est le domaine des tests respiratoires utilisant $\mathrm{le}^{13} \mathrm{C}$.

Le principe de ces tests respiratoires est simple. Il consiste à synthétiser un substrat en substituant par du ${ }^{13} \mathrm{C}$, un atome de carbone qui sera oxydé par un système biologique (enzyme) en dioxyde de carbone. Lors de l'oxydation de ce substrat marqué, il se forme du ${ }^{13} \mathrm{CO}_{2}$ qui est éliminé par la respiration. La mesure de l'enrichissement isotopique du $\mathrm{CO}_{2}$ des gaz expirés est un reflet de l'oxydation du substrat marqué et donc du fonctionnement du système enzymatique étudié. Sur ce principe se développent et se valident des tests non invasifs, non radioactifs, utilisant de faibles quantités de substrats et de faibles enrichissements. Les prélèvements sont très faciles (expiration dans un tube ou un sac) et seulement de faibles volumes sont prélevés (quelques $\mathrm{mL}$ ). Ces tests sont ainsi aisément réalisables sur toutes les catégories de patients : du nouveau-né à la personne très âgée.

Le domaine d'application majeur des tests respiratoires est celui de la gastro-entérologie [3]. Dans ce domaine, la CF-IRMS est en train de passer du laboratoire de recherche à celui de la « routine » de biologie médicale. En gastroentérologie, les tests respiratoires au ${ }^{13} \mathrm{C}$ permettent d'étudier des processus physio - pathologiques et métaboliques de façon indirecte. $\mathrm{Le}^{13} \mathrm{CO}_{2}$ est un métabolite terminal commun de tous les substrats utilisés et le traceur des processus étudiés. Le postulat doit être fait que le processus d'intérêt (absorption, métabolisme du traceur marqué) est l'étape limitante du processus entier étudié. À cause de la nature même des tests respiratoires qui impliquent une séquence de réactions et plusieurs pools métaboliques, les données qu'ils apportent sont souvent semi-quantitatives. Comme nous le verrons, ils peuvent remplacer des techniques invasives, onéreuses, difficiles à réaliser, par des processus simples et non dangereux.

\section{Infection par Helicobacter pylori}

La plus grande infection bactérienne au niveau mondial est due à Helicobacter pylori. Cette bactérie est reconnue comme le facteur causal de l'ulcère gastro-duodénal et a une part de responsabilité dans la survenue de certains cancers gastriques [4]. La caractéristique principale de cette bactérie qui colonise la muqueuse gastrique [5] est sa forte activité uréase [6]. Cette enzyme permet à Helicobacter pylori d'hydrolyser l'urée en dioxyde de carbone et ammoniac ce qui 
est un facteur permettant sa survie dans l'acidité gastrique mais est un des facteurs majeurs de sa pathogénicité. Le test respiratoire mis en jeu pour réaliser le diagnostic de la présence de Helicobacter pylori repose sur cette réaction d'hydrolyse. Il consiste à administrer au patient, par voie orale, une solution contenant de l'urée marquée par du ${ }^{13} \mathrm{C}$ et de mesurer après un certain temps l'enrichissement en ${ }^{13} \mathrm{C}$ du $\mathrm{CO}_{2}$ expiré. Si cet enrichissement mesuré par analyse isotopique s'élève au-dessus de la valeur basale mesurée avant la prise d'urée, il révèle la présence de Helicobacter pylori sur la muqueuse gastrique et signe un diagnostic positif. Si l'enrichissement en ${ }^{13} \mathrm{C}$ ne varie pas le test est négatif [7]. La méthode classique de diagnostic est une endoscopie au cours de laquelle des biopsies de l'antre et du fundus de l'estomac sont prélevées et étudiées par histologie pour observer la présence ou l'absence de Helicobacter pylori. Dans les divers pays qui utilisent déjà ce test, les gastroentérologues s'accordent pour estimer qu'une fraction non négligeable des endoscopies peut être remplacée par le test respiratoire [8].

Dans ce cas, la CF-IRMS a permis le développement et l'utilisation d'une méthode de diagnostic de la présence de Helicobacter pylori, non radioactive, non invasive, réalisable au cabinet du médecin grâce à un transport très aisé des échantillons de gaz au laboratoire, sans conditions spéciales. Pour ce qui est de l'analyse, Il existe sur le marché des analyseurs isotopiques dédiés (breath analyzers) hautement performants mesurant le ${ }^{13} \mathrm{C}$ par IRMS. Le test a d'excellentes spécificité et sensibilité, valeurs prédictives positives et négatives. Il est moins onéreux pour la collectivité que l'endoscopie et plus agréable et confortable pour le patient. Enfin, il est le seul test permettant de vérifier l'éradication de Helicobacter pylori après l'antibiothérapie. En effet, les anticorps anti Helicobacter pylori sécrétés par l'organisme, y demeurent plus de 12 à 18 mois ce qui rend les examens sérologiques inutilisables pour le contrôle de l'éradication de la bactérie [9].

\section{Évaluation de la fonction métabolique hépatique}

Un second secteur important d'applications des tests respiratoires en plein essor est celui de l'évaluation de la fonction métabolique hépatique. Le système cytochrome P450 est une superfamille d'enzymes métabolisant un grand nombre de médicaments et xénobiotiques et de nombreuses molécules endogènes (stéroïdes). Il est principalement localisé dans le foie. Cette superfamille se divise en familles et sous-familles ayant une plus ou moins grande spécificité de substrats. Des substrats marqués avec du ${ }^{13} \mathrm{C}$ en particulier sur des groupements $-\mathrm{N}-\mathrm{CH}_{3}$, peuvent être métabolisés par ces enzymes et le groupement méthyle marqué est oxydé en ${ }^{13} \mathrm{CO}_{2}$. A titre d'exemple, la $\left[3,{ }^{13} \mathrm{C}\right]$ caféine est métabolisée par le CYP1A2, l'aminopyrine et la méthacine sont aussi des substrats des CYP1A, l'érythromycine étant, quant à elle, un substrat du très important CYP3A4 [10]. Des tests respiratoires développés avec ces différentes molécules marquées par $\mathrm{du}{ }^{13} \mathrm{C}$ permettent d'explorer ces diverses isoformes du CYP450. Au niveau du foie la variété des substrats permet l'exploration systématique de l'hépatocyte. Ainsi les molécules citées plus haut permettent de mesurer l'activité microsomale [11], le ${ }^{13} \mathrm{C}$ galactose permet d'explorer la fonction cytosolique [12], l'acide ceto isocaproïque permet de suivre l'activité mitochondriale [13]. Le développement de l'analyse isotopique du $\mathrm{CO}_{2}$ a donc permis de développer des applications très importantes dans les domaines suivants :

- étude de la fonction hépatique chez des enfants souffrant de dermatite atopique [14], étude de la fonction hépatique chez des enfants soufrant de fibrose kystique [15] ;

- modification de la fonction hépatique par des médicaments : oméprazole [16], méthotrexate [17] ;

- utilisation de la phénacétine ${ }^{13} \mathrm{C}[18]$;

- étude de la maturation des CYP450 [19-20] ;

- étude de l'induction enzymatique du CYP 450 [21] ;

- étude d'inhibitions du CYP 450 [22].

\section{Exploration de la fonction exocrine du pancréas}

Un autre secteur d'application très intéressant est celui qui consiste à étudier la fonction exocrine du pancréas dans les pathologies de malabsorption et de maldigestion des graisses, comme cela est le cas chez les patients souffrant de fibrose kystique (mucoviscidose). L'enzyme dont l'activité est mesurée dans ce test est la lipase pancréatique qui hydrolyse les triglycérides dont les acides gras sont ensuite oxydés en générant du $\mathrm{CO}_{2}$ à partir de leur fonction carboxyle. Les substrats utilisés pour le développement de tests respiratoires sont des triglycérides à longue chaîne préférables à ceux à chaîne courte (trioctanoïne) [23] pour une meilleure spécificité. Ce sont essentiellement la tristéarine, la trioléïne ou des triglycérides mixtes comme le (1,3 distéaryl, 2-carboxyl- ${ }^{13} \mathrm{C}$ ) octanoyl glycérol [24]. Les tests classiques utilisés dans l'exploration de ces pathologies sont des balances entrée/sortie des graisses absorbées, des mesures de graisses, de la chymotrypsine et de l'élastase 1 fécales. Il a été montré que le test respiratoire aux triglycérides mixtes (TGM) permet de bien faire la distinction entre les populations de sujets sains et de patients atteints de mucoviscidose [25] et que dans des conditions d'utilisation parfaitement standardisées, il présente une bonne reproductibilité.

La comparaison du test utilisant la ${ }^{13} \mathrm{C}$ trioléïne avec l'analyse de la graisse fécale et le test à la sécrétine a montré une sensibilité de $91,7 \%$ et une spécificité de $86,7 \%$ pour la détection de la stéathorrée. Lembkle et al. ont ainsi conclu que chez les patients atteints de maladie pancréatique, le test à la trioléïne ${ }^{13} \mathrm{C}$ reflète le degré d'insuffisance de la lipase, indique une lipolyse décompensée et qu'il constitue une alternative pratique à l'analyse des graisses fécales [26]. Le test au $\mathrm{TGM}^{13} \mathrm{C}$ permet aussi de prévoir et de suivre la supplémentation en enzymes pancréatiques chez 
les enfants atteints de fibrose kystique et peut être utilisé pour optimiser le traitement [27].

\section{Mesure de la vidange gastrique}

La vidange gastrique n'est pas particulièrement aisée à étudier. C'est classiquement par scintigraphie que sont déterminés les paramètres de la vidange gastrique : temps de latence, temps de demi-vidange $\left(t^{1 / 2}\right)$, et coefficient de vidange gastrique. Un test respiratoire a été développé en utilisant l'acide ${ }^{13} \mathrm{C}$ octanoïque incorporé à un repas solide pour mesurer les paramètres de façon non radioactive et non invasive [28]. Cette détermination est particulièrement difficile chez les enfants et encore plus chez des nouveau-nés prématurés. Le test à l'acide ${ }^{13} \mathrm{C}$ octanoïque a pu être adapté à cette dernière population avec succès [29].

De nombreux autres tests sont utilisés dans d'autres secteurs de l'exploration fonctionnelle et du diagnostic.

L'acide ${ }^{13} \mathrm{C}$ palmitique permet d'étudier l'utilisation des acides gras [30].

Le ${ }^{13} \mathrm{C}$ xylose est utilisé pour le diagnostic de prolifération bactérienne intestinale [31].

Le bicarbonate ${ }^{13} \mathrm{C}$ permet de mesurer les dépenses énergétiques sur des intervalles de temps courts.

L'utilisation de la technique de l'eau doublement marquée conduit à l'estimation de la dépense énergétique sur des temps relativement longs. L'utilisation $\mathrm{du}{ }^{13} \mathrm{C}$ bicarbonate permet de mesurer la production de $\mathrm{CO}_{2}$ et la vitesse d'apparition du $\mathrm{CO}_{2}$. Ces mesures donnent un accès à l'estimation de la dépense énergétique sur des temps courts (quelques dizaines de minutes). Ce test a été utilisé chez l'animal et avec succès chez les enfants et les nouveau-nés prématurés [32-33].

Le dernier test que nous citerons pour clore cette liste non exhaustive est celui qui utilise un substrat marqué à la fois avec du ${ }^{13} \mathrm{C}$ et du ${ }^{15} \mathrm{~N}$. Ce test destiné à mesurer le temps de transit oro-coecal, utilise en effet le lactose $\left[{ }^{13} \mathrm{C}-{ }^{15} \mathrm{~N}\right]$ uréide. Il met en jeu la détermination de l'enrichissement du ${ }^{13} \mathrm{CO}_{2}$ et de l'azote ${ }^{15} \mathrm{~N}$ urinaire par GC-C-IRMS permet de remplacer le classique test au lactulose qui manque à la fois de sélectivité et de spécificité [34].

\section{Nouvelles technologies}

Le second domaine dans lequel la spectrométrie de masse isotopique apporte des améliorations significatives est celui des explorations utilisant de très faibles quantités d'analyte, des niveaux très bas de marquage et l'utilisation de substrats possédant simplement des enrichissements au niveau naturel. Dans ces domaines la spectrométrie des masses isotopiques en flux continu peut aussi se substituer à de technologies préexistantes et en particulier au couplage chromatographie en phase gazeuse-spectrométrie de masse organique conventionnelle et permet d'élaborer des proto- coles de recherche nouveaux et d'accéder à des niveaux de performance améliorés.

\section{Utilisation de micro-quantités d'analyte}

Très souvent les études biomédicales sont limitées par la dimension des pièces analytiques et des quantités d'analytes dont peut disposer le laboratoire pour les déterminations quantitatives. La possibilité de ne disposer que de très faibles quantités d'analyte amène à changer de technologie d'analyse et par exemple de passer du couplage GC-MS à la GC - C-IRMS. Pont et al. [35] étudiant la cinétique des lipoprotéines ont comparé les performances respectives de la GC-MS et de la GC-C-IRMS. Ces auteurs ont ainsi pu mesurer la richesse isotopique en ${ }^{13} \mathrm{C}$ du cholestérol et de la leucine extraits des VLDL (very low density lipoproteins) et des LDL (low density lipoproteins) après administration à des patients, de faibles quantités de $\left[3,4\left({ }^{13} \mathrm{C}\right)\right.$ cholestérol $]$ et $\left[1-{ }^{13} \mathrm{C}\right.$-leucine]. Les résultats ont montré que la GC-C-IRMS donne des résultats précis et exacts sur un large intervalle d'enrichissement $(-20,+700 \%$ ) à partir de spécimens analytiques de 0,1 à $10 \mathrm{ng}$ et que la spectrométrie de masse isotopique est plus exacte et plus reproductible que la GC-MS classique.

La possibilité de travailler sur des quantités très faibles d'échantillons permet de réaliser des études sur des pièces d'exérèse collectées lors de simples endoscopies plutôt que sur des pièces plus volumineuses nécessitant une intervention chirurgicale plus sévère. C'est ainsi, par exemple, que la synthèse protéique dans le cancer rectal humain a pu être étudiée in situ, par perfusion de $\left[{ }^{13} \mathrm{C}\right.$-leucine $]$ suivie d'une mesure de l'enrichissement isotopique de la leucine incorporée dans les protéines tumorales, par GC-C-IRMS [36]. Les méthodes analytiques utilisées antérieurement nécessitaient la collecte d'une pièce chirurgicale. L'utilisation de la GC-C-IRMS permet de travailler à partir de micro-biopsies prélevées lors d'une rectosigmoïdoscopie conventionnelle. L'utilisation de méthodologies mettant en jeu des microgénérateurs de $\mathrm{CO}_{2}$ permet encore d'augmenter les performances analytiques. Les microgénérateurs de $\mathrm{CO}_{2}$ sont des systèmes chimiques, biochimiques (enzymatiques - cellulaires) permettant, à partir d'un substrat marqué au ${ }^{13} \mathrm{C}$ de générer de micro-quantités de $\mathrm{CO}_{2}$. Celui-ci est ensuite analysé pour son contenu en ${ }^{13} \mathrm{C}$ avec grandes sensibilité et précision. Dans le cas des aminoacides comme la leucine, lors de la détermination du marquage par GC-C-IRMS, comme dans l'exemple précédent, le marquage en $\mathrm{C} 1$ est dilué par le reste du squelette carboné qui brûlé dans le four de combustion génère du $\mathrm{CO}_{2}$. La technologie des microgénérateurs de $\mathrm{CO}_{2}$ permet d'utiliser la méthode de Van Slyke qui consiste à faire réagir la ninhydrine sur l'aminoacide marqué à l'aide de ${ }^{13} \mathrm{C}$ sur le groupement carbonyle. Celui-ci est alors décarboxylé spécifiquement et ce seul atome de carbone génère le $\mathrm{CO}_{2}$ qui est analysé. Ainsi à partir d'une petite biopsie, il est possible d'extraire les protéines, de les hydrolyser, de séparer les acides aminés et de faire réagir la 
fraction d'intérêt avec la ninhydrine. La détermination finale de l'enrichissement en ${ }^{13} \mathrm{C}$ se fera sur ce $\mathrm{CO}_{2}$ issu de cette réaction spécifique. Il est ainsi possible de déterminer de faibles enrichissements en ${ }^{13} \mathrm{C}$ à partir de très petits échantillons analytiques.

L'utilisation de la technologie des microgénérateurs peut être couplée à l'utilisation de traceurs marqués sur des sites spécifiques différents du même substrat afin de permettre l'étude de mécanismes réactionnels ou de voies métaboliques.

L'activation de la NADPH oxydase du polynucléaire est réalisée après interaction entre certains récepteurs membranaires et de nombreux stimuli (acétate de phorbol myristate PMA, bactéries opsonisées etc.). Le NADPH utilisé par la NADPH oxydase pour produire des ions superoxyde provient de la voie des hexoses monophosphates. Cette voie est intra-cytosolique, à partir d'une molécule de glucose 6 phosphate provenant du glucose, deux molécules de NADPH sont générées avec production d'une molécule de $\mathrm{CO}_{2}$. L'utilisation de molécules de glucose marquées soit en 1, en 2 ou en 6 a permis d'explorer plusieurs voies métaboliques lors de l'activation des polynucléaires. Incubé avec les polynucléaires, le glucose pourra être métabolisé par différentes voies [37]. Le site 1 sera utilisé par la voie des hexoses monophosphates, le carbone 2 renseignera sur un recyclage $\mathrm{du}$ glucose, l'utilisation du site 6 marquera la glycolyse. La mesure de l'enrichissement en ${ }^{13} \mathrm{C}$ et de la production de ${ }^{13} \mathrm{CO}_{2}$ lors du stress oxydatif, par dilution isotopique inverse du $\mathrm{CO}_{2}$ produit à partir des différents isotopomères du ${ }^{13} \mathrm{C}$ glucose métabolisé par $5.10^{5}$ cellules stimulées par du PMA, a permis de montrer la prépondérance du shunt des hexoses monophosphates, le recyclage du glucose et l'absence de glycolyse lors de l'activation des polynucléaires neutrophiles. Les avantages de la CF-IRMS sont évidents si l'on compare aux premiers travaux qui utilisaient les traceurs au ${ }^{14} \mathrm{C}$. Le nombre de cellules utilisées avec le marquage au ${ }^{13} \mathrm{C}$ et l'IRMS est 100 fois inférieur à celui nécessaire lors des études conduites avec le ${ }^{14} \mathrm{C}$. De plus, le marquage spécifique sur les sites 1,2 et 6 apporte des renseignements capitaux quant aux voies métaboliques utilisées. Il est de plus possible par la méthode de dilution isotopique inverse de calculer le volume de $\mathrm{CO}_{2}$ généré par les cellules lors de leur activation.

\section{Coût des substrats marqués}

Une des limitations à l'usage des méthodes classiques de traçages avec des molécules marquées par des isotopes stables lors des études métaboliques réside dans la quantité de traceur à utiliser si les pools métaboliques sont importants et/ou si le turn over des substrats est rapide. Il s'ensuit un coût élevé lié à cette quantité importante de traceur.

Il est maintenant possible de mettre en œuvre ses études grâce à l'IRMS et à l'utilisation de très faibles quantités de traceurs qui de plus utilisés en faibles quantités ne perturbent pas les pools métaboliques.

La glutamine est essentielle pour les cellules qui présentent un taux de renouvellement ou de réplication élevé comme les entérocytes ou les lymphocytes. L'utilisation de glutamine marquée au ${ }^{13} \mathrm{C}$ a été limitée par le coût prohibitif du traceur et la difficulté de mesurer de faibles quantités et enrichissements par GC-MS. La GC - IRMS a permis de mettre en place des études métaboliques avec ce traceur et autorise aussi la détermination de faibles enrichissements $(<0,06 \mathrm{~mol} \%$ excess) avec une précision meilleure que $5 \%$ [38]. Les mêmes limitations sont apparues lors de l'étude de la cinétique de l'urée dont les taux de production et d'élimination sont très importants. Là encore, les quantités de traceur marqué à utiliser et le coût du marquage ont longtemps entravé les études des paramètres cinétiques de l'urée à une large échelle [39]. L'utilisation de l'urée marquée au ${ }^{13} \mathrm{C}$ sa détermination dans le plasma par microgénération de $\mathrm{CO}_{2}$ sous l'action d'uréase et la mesure de l'enrichissement en ${ }^{13} \mathrm{C}$ par IRMS du $\mathrm{CO}_{2}$ produit $(0,02 \mathrm{~mol} \%$ excess $)$ a ainsi permis la détermination du volume de distribution, du taux de production et de la clairance de l'urée.

\section{Utilisation de substrats naturellement enrichis}

En poussant plus loin les exigences techniques et les qualités analytiques, il est possible de concevoir l'utilisation de traceurs possédant de très faibles enrichissements et donc la possibilité de s'adresser à des traceurs naturellement enrichis. Il est bien connu que selon la provenance botanique (plantes C3 ou plantes $\mathrm{C} 4$ ) et donc selon les voies métaboliques de la photosynthèse, les hydrates de carbone synthétisés par les plantes ne présentent pas tous la même richesse isotopique en ${ }^{13} \mathrm{C}$. Ainsi l'absorption de glucose de maïs ou de betterave conduira à rejeter du $\mathrm{CO}_{2}$ produit par le métabolisme qui sera respectivement de 1,0885 At $\%$ et 1,0822 $\mathrm{At} \%$. Il s'avère que la richesse isotopique naturelle des substrats peut être suffisante pour conduire des études de traçage métabolique. Les nouveau-nés nécessitent de l'acide arachidonique pour la croissance de leurs tissus. Il est ainsi important de connaître avec quel rendement il leur est possible de synthétiser l'acide arachidonique à partir de l'acide linoléique contenu dans leur alimentation. L'équipe allemande de Demmelmair [40] a remplacé la diète de ces enfants (alimentation par le lait maternel) par de l'huile de maïs comme seule source de matière grasse. L'acide linoléique de maïs a un contenu en ${ }^{13} \mathrm{C}$ supérieur à celui de toutes les graisses utilisées communément en Europe. De plus cette huile ne contient pas d'acide arachidonique. Quatre jours après le changement de régime, l'acide arachidonique des enfants montrait un enrichissement augmenté de $2,7 \%$ prouvant ainsi une synthèse endogène active de l'acide arachidonique à partir de l'acide linoléique chez ces nouveau-nés. 
Une telle étude montre que la CF-C-IRMS permet d'utiliser des molécules naturellement enrichies, de travailler avec des taux de marquage extrêmement faibles et avec une sensibilité élevée et de conduire des protocoles parfaitement éthiques dans des populations de sujets particulièrement protégées, ici le nouveau-né. Du lait naturellement enrichi a, de la même façon, permis la production de lactose pour des études métaboliques. Des protéines d'œuf de poules dont l'alimentation était supplémentée avec $0,2 \%$ de ${ }^{13} \mathrm{C}$ leucine ont été enrichies à 1,3371 At\% ce qui est suffisant pour des études portant sur l'assimilation et la cinétique métabolique de ces protéines chez l'homme [41].

\section{Conclusion}

Le développement des technologies de spectrométrie de masse a permis à ces dernières de gagner les laboratoires de recherche du domaine biomédical et de prestation de service de biochimie clinique avec l'installation de systèmes de couplage GC-MS et LC-MS. Depuis ces toutes dernières années, les progrès de l'analyse isotopique et le développement de technologies utilisant les isotopes stables ouvrent le domaine de la recherche biomédicale et du diagnostic à ces technologies tout d'abord développées et utilisées dans le domaine des géosciences et de l'environnement. Cette évolution technologique permet d'accéder à de nouveaux territoires de recherche, soit en augmentant l'efficacité des protocoles, soit en permettant de réaliser des protocoles jusqu'alors impossibles ou trop complexes ou trop onéreux. Elle permet en outre d'accéder à de nouvelles populations de patients extrêmement protégés comme la femme enceinte et le nouveau-né. Les domaines de la nutrition, du métabolisme, de la pharmacologie et du diagnostic sont en train de bénéficier de cet apport nouveau de technologie et à en juger par l'accroissement du rythme des publications scientifiques portant sur l'analyse isotopique dans le domaine des biosciences, l'avenir est extrêmement prometteur.

\section{Références}

1. Foster, A. B. Adv. Drug. Res. 1985, 14, 1-36.

2. Benchekroun, Y.; Dautraix, S.; Desage, M.; Brazier, J. L. Eur. J. Drug Metab. Pharmacokin. 1997, 22, 127-133.

3. Swart, G. R.; Van den Berg, J. W. Scand. J. Gastroenterol. 1998, 225, 13-18.

4. Cats, A.; Meuwissen; Forman, D.; Craanen, M. E.; Kuipers, E. J. Eur. J. Gastroenterol. Hepatol. 1998, 10, 447-450.

5. Clyne, M.; Drumm, B. Can. J. Gastroenterol. 1997, 11, $243-$ 248.

6. Hawtin, P. R.; Sracey, A. R.; Newell, D. G. J. Gen. Microbiol. 1990, 136, 1995-2000.

7. Mion, F.; Dewcluze, H.; Rousseau, M.; Berger, F.; Brazier, J. L.; Minaire, Y. Gastroenterol. Clin. Biol. 1994, 18, 1106-1111.

8. Hunt, R.; Thomson, A. B. Can. J. Gastroenterol. 1998, 12, 3141.

9. Fallone, C. A.; Wild, G. E.; Goresky, C. A.; Barkun, A. N. Can. J. Gastroenterol. 1995, 9, 105-111.
10. Wagner, D. Clin. Pharm. Therap. 1998, 64, 129.

11. Mion, F.; Queneau, P.; Rousseau, M.; Brazier, J. L.; Paillard, P.; Minaire, Y. Hepato-gastroenterology 1995, 42, 931-938.

12. Berry, G. T.; Nissim, I.; Mazur, A. T.; Elsas, L. J.; Singh, R. H.; Klein, P. D.; Gibson, J. B.; Lin, Z.; Segal, S. Bioch. Mol. Med. 1995, 56, 158-165.

13. Lauterburg, B. H.; Grattagliano, I.; Gmur, R.; Stalder, M.; Hildebrand, P. J. Lab. Clin. Med. 1995, 125, 378-383.

14. Likura, Y.; Iwasaki, A.; Tsubaki, T.; Akasawa, A.; Onda, T.; Katsunuma, T.; Miura, K.; Ebisawa, M.; Saito, H.; Koya, N. Int. Arch. Allerg. Immunol. 1995, 107, 189-193.

15. Parker, A. C.; Pritchard, P.; preston, T.; Smyth, R. L.; Choonara, I. Arch. Diseases. Child. 1997, 77, 239-241.

16. Rost, K. L.; Brosicke, H.; Brockmoller, J.; Scheffler, M.; Helge, H.; Roots, I. Clin. Pharm. Therap. 1992, 52, 170-180.

17. Guitton, J.; Souillet, G.; Riviere, J. L.; Gerard, F.; Guilluy, R.; Brazier, J. L. Eur. J. Drug Metab. Pharmacokin. 1994, 19, $119-124$

18. Kajiwara, M.; Okazaki, T.; Ida, K.; Marumi, S.; Hirose, M.; Ijicji, M.; Abel, T.; Hirano, S.; Limura, M. Chem. Pharm. Bul. 1996, 44, 1258-1260.

19. Pons, G.; Blais, J. C.; Rey, E.; Plissonnier, M.; Richard, M. O.; Carrier, O.; D’Athis, P.; Moran, C.; Badoual, J.; Olive, G. Pediat. Res. 1988, 23, 632-636.

20. Krueger, N.; Helge, H.; Neubert, D. Develop. Pharmacol. Therap. 1991, 16, 164-175.

21. Guilluy R.; Brazier J. L.; Billion Rey F. J. Chromatogr. 1991, 562, 341-350.

22. Parker, A. C.; Preston, T.; Heaf, D.; Kitteringham, N. R.; Choonara, I. Brit. J. Clin. Pharmacol, 1994, 38, 573-576.

23. Hoshi, J.; Nishida, H.; Yasui, M.; Ohishi, M.; Takahashi, M. Acta. Ped. Jap. 1992, 34, 374-677.

24. Loser, C.; Brauer, C.; Aygen, S.; Hennemann, O.; Folsch, U. R. Scand. J. Gastoenterol. 1998, 33, 327-334.

25. Swart, G. R.; Baartman, E. A.; Wattimena, J. L.; Rietveld, T.; Overbeek, S. E.; van den Berg, J. W. Digestion 1997, 58, 415420.

26. Lembcke, B.; Braden, B.; Caspary, W. F. Gut 1996, 39, 668674.

27. Amarri, S.; Harding, M.; Coward, W. A.; Evans, T. J.; Weaver, L. T. Arch. Diseas. Child. 1997, 76, 349-351.

28. Choi, M. G.; Camillieri, M.; Burton, D. D.; Zinsmeister, A. R.; Nair, K. S. Gastroenterology 1997, 112, 1155-1162.

29. Veereman-Wauters, G.; Ghoos, Y.; van der Schoor, S.; Maes, B.; Hebbalkar, N.; Devlieger, H.; Eggermont, E. J. Pediat. Gastroenterol. Nut. 1996, 23, 111-117.

30. Jones, A. E.; Murphy, J. L.; Stolinski, M.; Wooton, S. A. Eur. J. Clin. Nut. 1998, 52, 22-28.

31. Dellert, S. F.; Nowicki, M. J.; Farrell, M. K.; Delente, J.; Heubi, J. E. J. Pediat. Gastroenterol. Nut. 1997, 25, 153-158.

32. Horswill, C. A.; Zipf, W. B.; Kien, C. L.; Med. Sci. Sports Exer. 1997, 29, 1263-1268.

33. Kien, C. L.; McClead, R. E. J. Parent. Ent. Nutr. 1996, 20, 389-393.

34. Wultzke, K. D.; Heine, W. E.; Plath, C.; Leitzmann, P.; Radke, M.; Mohr, C.; Richter, I.; Gulzow, H. U.; Hobusch, D. Eur . J. Clin. Nutr. 1997, 51, 11-19.

35. Pont, F.; Duvillard, L.; Maugeais, C.; Athiqs, A.; Persegol, L.; Gambert, P.; Vergers, B. Anal. Biochem. 1997, 248, 277-287. 
36. Hartl, W. H.; Demmelmair, H.; Jauch, K. W.; Schmidt, H. L.; Koletzco, B.; Schildberg, F. W. AM. J. Physiol. 1997, 272, E796-802.

37. Lamrini, R.; Lacan, P.; Francina, A.; Guilluy, R.; Brazier, J. L. Rapid Com. Mass. Spectrom. 1995, 1017-1020.

38. Menand, C.; Pouteau, E.; Marchini, S.; Maugere, P.; Krempf, M.; Darmaun, D. J. Mass Spectrom. 1997, 32, 1097-2009.
39. Kloppenburg, W. D.; Wolthers, B. G.; Stellaard, F.; Elzinga, H.; Tepper, T.; de Jong, P. E.; Huisman, R. M. Clin . Sci. 1997, 93, 73-80.

40. Demmelmair, H.; von Schenck, U.; Behrendt, E.; Sauerwald, T.; Koletzko, B. J. Pediat. Gastroenterol. Nutr. 1995, 21, 3136.

41. Evenepoel, P.; Hiele, M.; Luypaerts, B.; Buyse, J.; Decuypere, E.; Ruitgeerts, P.; Ghoos, Y. J. Nutrition. 1997, 127, 327-331. 